\title{
BOREL CIRCLE SQUARING
}

\author{
ANDREW S. MARKS AND SPENCER T. UNGER
}

\begin{abstract}
We give a completely constructive solution to Tarski's circle squaring problem. More generally, we prove a Borel version of an equidecomposition theorem due to Laczkovich. If $k \geq 1$ and $A, B \subseteq \mathbb{R}^{k}$ are bounded Borel sets with the same positive Lebesgue measure whose boundaries have upper Minkowski dimension less than $k$, then $A$ and $B$ are equidecomposable by translations using Borel pieces. This answers a question of Wagon. Our proof uses ideas from the study of flows in graphs, and a recent result of Gao, Jackson, Krohne, and Seward on special types of witnesses to the hyperfiniteness of free Borel actions of $\mathbb{Z}^{d}$.
\end{abstract}

\section{INTRODUCTION}

In 1925, Tarski posed the problem of whether a disk and square of the same area in the plane are equidecomposable by isometries $[\mathrm{T}$. That is, can a disk be partitioned into finitely many pieces which can be rearranged by isometries to partition a square of the same area? This problem became known as Tarski's circle squaring problem. In contrast to the Banach-Tarski paradox in $\mathbb{R}^{3}$, a theorem of Tarski (see [W]) implies that any two Lebesgue measurable sets in $\mathbb{R}^{2}$ that are equidecomposable by isometries must have the same Lebesgue measure, even when the pieces used in the equidecomposition are allowed to be nonmeasurable. Thus, the requirement that the circle and the square have the same area is necessary.

The idea of comparing the measure of sets by partitioning them into congruent pieces has a long history, dating back in some form to Euclid. The well known Wallace-Bolyai-Gerwien theorem states that two polygons in $\mathbb{R}^{2}$ have the same area if and only if they are dissection congruent, that is, equidecomposable by polygonal pieces where we may ignore boundaries. Hilbert's third problem asked whether any two polyhedra of the same volume are dissection congruent. Dehn famously gave a negative answer to this problem.

Early work on Tarski's circle squaring problem established the nonexistence of certain types of equidecompositions. Dubins, Hirsch, and Karush DHK introduced the notion of scissors congruence in $\mathbb{R}^{2}$, considering equidecompositions using pieces whose boundaries consist of a single Jordan curve. They showed that a disk in $\mathbb{R}^{2}$ is scissors congruent to no convex set other than translates of itself. So Tarski's circle squaring problem has a negative answer for this restrictive type of equidecomposition. Gardner [G] also showed that Tarski's circle squaring problem cannot be solved using any locally discrete subgroup of isometries.

Laczkovich answered Tarski's question positively in 1990 [L90, using only translations in his equidecomposition. In 1992, he improved this result to give a very general sufficient condition for when two bounded sets in $\mathbb{R}^{k}$ of the same Lebesgue

Date: June 14, 2017.

The first author was partially supported by NSF grant DMS-1500974. 
measure are equidecomposable by translations. If $X \subseteq \mathbb{R}^{k}$, then we let $\partial X=$ $\operatorname{cl}(X) \backslash \operatorname{int}(X)$ indicate its boundary and $\Delta(X)$ be the upper Minkowski dimension of $X$ (see [L92]). Let $\lambda$ be Lebesgue measure.

Theorem 1.1 ([L92, Theorem 3]). Suppose $k \geq 1$ and suppose $A, B \subseteq \mathbb{R}^{k}$ are bounded sets such that $\lambda(A)=\lambda(B)>0, \Delta(\partial A)<k$, and $\Delta(\partial B)<k$. Then $A$ and $B$ are equidecomposable by translations.

Laczkovich's proofs in [290] and [292] are nonconstructive and use the axiom of choice. It remained an open problem whether such equidecompositions could be done constructively. In WW, Appendix C, Question 2.a], Wagon made this question precise by asking whether Tarski's circle squaring problem could be solved using Borel pieces. Recall that the Borel sets are the smallest collection of sets obtained by starting with the open sets and closing under the operations of countable union, countable intersection, and complementation.

In a recent breakthrough, Grabowski, Máthé, and Pikhurko GMP showed that Tarski's circle squaring problem can be solved using Lebesgue measurable or Baire measurable pieces, by proving a version of Theorem 1.1 for Lebesgue measurable/Baire measurable equidecompositions. Their proof is also non-constructive since it uses the axiom of choice to construct the equidecomposition on a null/meager set. However, their result gave strong evidence that a constructive solution to Tarski's circle squaring problem might exist, since it showed that there cannot be any measure-theoretic or Baire category obstruction.

In this paper, we answer Wagon's question [W, Appendix C, Question 2.a] and give a completely constructive solution to Tarski's circle squaring problem. More generally, we prove a Borel version of Laczkovich's Theorem 1.1. This generalizes the results of GMP. It also provides a "Borel solution" to Hilbert's third problem: any two bounded Borel sets in $\mathbb{R}^{k}$ with "small boundary" have the same measure if and only if they are translation equidecomposable using Borel pieces:

Theorem 1.2. Suppose $k \geq 1$ and suppose $A, B \subseteq \mathbb{R}^{k}$ are bounded Borel sets such that $\lambda(A)=\lambda(B)>0, \Delta(\partial A)<k$, and $\Delta(\partial B)<k$. Then $A$ and $B$ are equidecomposable by translations using Borel pieces.

The pieces that we use in our equidecomposition are quite simple. Let $\boldsymbol{\Sigma}_{1}^{A, B}$ be the collection of all open balls in $\mathbb{R}^{k}$, translates of $A$, and translates of $B$. Then inductively, let $\mathcal{B}_{n}^{A, B}$ be all finite Boolean combinations of $\boldsymbol{\Sigma}_{n}^{A, B}$ sets, and let $\boldsymbol{\Sigma}_{n+1}^{A, B}$ be all countable unions of sets in $\mathcal{B}_{n}^{A, B}$. If $A$ and $B$ are $\boldsymbol{\Sigma}_{m}^{0}$ in the usual Borel hierarchy, then clearly every set in $\boldsymbol{\Sigma}_{n}^{A, B}$ is $\boldsymbol{\Sigma}_{n+m-1}^{0}$. The pieces we use in our equidecomposition are sets in $\mathcal{B}_{4}^{A, B}$ (see Section 17). If $A$ and $B$ are a disk and a square of the same area in $\mathbb{R}^{2}$, then it is easy to see that $A$ and $B$ are not equidecomposable using set in $\mathcal{B}_{1}^{A, B}$, since $A$ and $B$ are not scissors congruent.

A key idea in our proof is to use flows in infinite graphs as an intermediate step towards constructing equidecompositions. Under the hypotheses of Theorem 1.2 in Section 4 we give an explicit and simple construction of a bounded "Borel flow" between $A$ and $B$. Laczkovich's discrepancy estimates from [L92] - the central ingredient in the proof of Theorem 1.1 - are used to show the convergence of this construction.

Another important tool in our proof comes from the theory of orbit equivalence and Borel equivalence relations. In particular, we use a result of Gao, Jackson, 
Krohne, and Seward, about special types of witnesses to the hyperfiniteness of free Borel actions of $\mathbb{Z}^{d}$ (see Theorem 5.5). Their theorem is part of an ongoing research program to understand the complexity of actions of amenable groups in descriptive set theory and ergodic theory. It builds on the result due to Weiss that every free Borel action of $\mathbb{Z}^{d}$ is hyperfinite, and more recent work of Gao-Jackson GJ] (see also $[\mathrm{SS}$ ). Gao, Jackson, Krohne, and Seward's theorem is announced in GJKS, but has not yet appeared, and so we include a proof of their result in Appendix A for completeness. (This proof is different from their forthcoming proof). So our paper is essentially self-contained except for our use of Laczkovich's discrepancy estimates.

In Section 5 we use this hyperfiniteness witness to turn our real-valued Borel flow between $A$ and $B$ into an integer-valued flow. This step in our proof also relies on the integral flow theorem which is a corollary of the Ford-Fulkerson proof of the max-flow min-cut theorem. The last step in our proof in Section 6 uses this integer valued flow to define a Borel equidecomposition from $A$ to $B$.

These ideas are very different from the work of GMP. The tools they use are quite specific to the measurable and Baire measurable settings and cannot easily be adapted to prove Theorem 1.2

The authors would like to thank Anton Bernshteyn, Clinton Conley, Steve Jackson, Alekos Kechris, Igor Pak, Robin Tucker-Drob, and Brandon Seward for helpful discussions.

\section{Preliminaries}

If $a: \Gamma \curvearrowright X$ is an action of a group $\Gamma$ on a set $X$, then $A, B \subseteq X$ are said to be a-equidecomposable if there exist a partition $\left\{A_{1}, \ldots, A_{n}\right\}$ of $A$ and group elements $\gamma_{1}, \ldots, \gamma_{n} \in \Gamma$ such that $\gamma_{1} \cdot A_{1}, \ldots, \gamma_{n} \cdot A_{n}$ is a partition of $B$. Similarly we say that $A, B \subseteq X$ are a-equidecomposable using Borel pieces if there exist a partition $\left\{A_{1}, \ldots, A_{n}\right\}$ of $A$ into Borel sets and group elements $\gamma_{1}, \ldots, \gamma_{n} \in \Gamma$ such that $\gamma_{1} \cdot A_{1}, \ldots, \gamma_{n} A_{n}$ is a partition of $B$ into Borel pieces.

Suppose $A, B \subseteq \mathbb{R}^{k}$ are bounded, and we wish to show that $A$ and $B$ are equidecomposable by translations using Borel pieces. By scaling and translating $A$ and $B$, we may assume that $A, B \subseteq[0,1 / 2)^{k}$ which is a subset of the $k$-torus $\mathbb{T}^{k}=\mathbb{R}^{k} / \mathbb{Z}^{k}$ which we identify with $[0,1)^{k}$. Then it is clear that any equidecomposition by translations between $A$ and $B$ in $\mathbb{T}^{k}$ can also be done in $\mathbb{R}^{k}$ using the same set of pieces. This idea was used by Laczkovich [L90]. We will work in $\mathbb{T}^{k}$ throughout the paper and show that $A$ and $B$ are equidecomposable by translations using Borel pieces in $\mathbb{T}^{k}$.

$\mathbb{T}^{k}$ inherits both its topology and abelian group structure from $\mathbb{R}^{k}$. We let $\lambda$ be Haar measure on $\mathbb{R}^{k} / \mathbb{Z}^{k}$ which we can identify with Lebesgue measure on the fundamental domain $[0,1)^{k}$. If $F \subseteq \mathbb{T}^{k}$ is finite and $A \subseteq \mathbb{T}^{k}$ is $\lambda$-measurable, then the discrepancy of $F$ relative to $A$ is

$$
D(F, A)=|| F \cap A|/| F|-\lambda(A)| .
$$

Given $\mathbf{u}=\left(u_{1}, \ldots, u_{d}\right) \in\left(\mathbb{T}^{k}\right)^{d}$, let $a_{\mathbf{u}}$ be the action of $\mathbb{Z}^{d}$ on $\mathbb{T}^{k}$ defined by

$$
\left(n_{1}, \ldots, n_{d}\right) \cdot a_{\mathbf{u}} x=n_{1} u_{1}+\ldots+n_{d} u_{d}+x
$$

for $\left(n_{1}, \ldots, n_{d}\right) \in \mathbb{Z}^{d}$ and $x \in \mathbb{T}^{k}$. Let

$$
R_{N}=\left\{\left(n_{1}, \ldots, n_{d}\right) \in \mathbb{Z}^{d}: 0 \leq n_{i}<N \text { for every } i \leq d\right\}
$$


and let the image of this set under the action $a_{\mathbf{u}}$ be

$$
F_{N}\left(x, a_{\mathbf{u}}\right)=R_{N} \cdot a_{\mathbf{u}} x .
$$

Laczkovich proved the following crucial estimate on the discrepancies of these sets, using ideas from Diophantine approximation, and building on work of Schmidt $[\underline{S}$ and Niederreiter and Wills [NW].

Lemma 2.1 (Laczkovich [L92, Proof of Theorem 3], see also GMP, Lemma 6]). Suppose $A \subseteq \mathbb{T}^{k}$ is measurable, $\Delta(\partial A)<k$ and $\lambda(A)>0$. Let $d$ be such that $d>2 k /(k-\Delta(\partial A))$. Then for almost every $\mathbf{u} \in\left(\mathbb{T}^{k}\right)^{d}$, there is an $\epsilon>0$ and $M>0$ such that for every $x \in \mathbb{T}^{k}$ and $N>0$,

$$
D\left(F_{N}\left(x, a_{\mathbf{u}}\right), A\right) \leq M N^{-1-\epsilon} .
$$

The variables $u,\left(x_{1}, \ldots, x_{d}\right)$, and $\eta$ in L92 correspond to our $x$, $\mathbf{u}$, and $\epsilon$, respectively.

Though we will not need this observation for our proof, we remark that the order of the quantifiers over $\mathbf{u}$ and $A$ can be reversed here. Almost every $\mathbf{u} \in\left(\mathbb{T}^{k}\right)^{d}$ satisfies the lemma for every measurable $A \subseteq \mathbb{T}^{k}$. This follows from Laczkovich's argument.

By a graph $G$ on a set $V$, we mean a (simple undirected) graph with vertex set $V$, so the edge relation of $G$ will be a symmetric irreflexive relation on $V$. If $G$ is a graph on a vertex set $V$ and $x \in V$, then we write $[x]_{G}$ for the set of vertices in the same connected component as $x$. We let $d_{G}$ be the graph metric on the vertex set of $G$. We will write $d$ instead of $d_{G}$ when the graph $G$ is clear from context. Let $N_{G}(x)$ be the set of neighbors of $x$, so $N_{G}(x)=\left\{y \in V: d_{G}(x, y)=1\right\}$. We will write $N(x)$ when the graph is clear. A graph $G$ is said to be locally finite if $N_{G}(x)$ is finite for every vertex $x$ of $G$. If $U \subseteq V$, then we let $G\lceil U$ be the induced subgraph of $G$ on the set $U$. If $F$ is a set of edges of $G$, we let $G-F$ be the subgraph of $G$ obtain by removed the edges in $F$.

If $a: \mathbb{Z}^{d} \curvearrowright X$ is an action of $\mathbb{Z}^{d}$ on a set $X$, let $G_{a}$ be the graph with vertex set $X$ where there is an edge from $x$ to $y$ if $\gamma \cdot x=y$ for some $\gamma \in \mathbb{Z}^{d}$ with $|\gamma|_{\infty}=1$. Here $\left|\left(\gamma_{1}, \ldots, \gamma_{d}\right)\right|_{\infty}=\sup _{i}\left|\gamma_{i}\right|$ is the sup norm. The bulk of our proof is establishing the existence of certain types of flows (defined in Section 3) on the graph $G_{a_{\mathbf{u}}}$ where $a_{\mathbf{u}}$ is as in Lemma 2.1.

Recall that a standard Borel space is a set $X$ equipped with a $\sigma$-algebra generated by a Polish (separable, completely metrizable) topology on $X$. The space $\mathbb{T}^{k}$ equipped with its Borel sets is an example of a standard Borel space. If $X$ is a standard Borel space and $n>0$ then we equip $X^{n}$ with the standard Borel space arising from the product topology on $X^{n}$. We will use $[X]^{<\infty}$ to note the standard Borel space of all finite subsets of $X$. Here we use the standard Borel structure where $B \subseteq[X]^{<\infty}$ is Borel if for every $n,\left\{\left(x_{1}, \ldots, x_{n}\right) \in X^{n}:\left\{x_{1}, \ldots, x_{n}\right\} \in B\right\}$ is a Borel subset of $X^{n}$.

In some of our proofs, we will need to make an arbitrary choice between finitely many elements of some standard Borel space $X$. In this situation we will fix some Borel linear ordering $<$ on $X$, and choose the <-least element. Note that every standard Borel space admits a Borel linear ordering (one can see this using the isomorphism theorem for standard Borel spaces and the fact that the usual linear ordering on $\mathbb{R}$ is Borel $\left[\mathrm{K}\right.$, Theorem 15.6]). In the case when $X=\mathbb{T}^{k}$, we may simply use the lexicographic ordering on $[0,1)^{k}$. 
A Borel graph is a graph whose vertices are the elements of a standard Borel space $X$, and whose edge relation is Borel as a subset of $X \times X$. For a recent survey of the theory of Borel graphs, see [KM]. If $\mathbf{u} \in\left(\mathbb{T}^{k}\right)^{d}$, then the graph $G_{a_{\mathbf{u}}}$ is an example of a Borel graph; since the action $a_{\mathbf{u}}$ is continuous, the edge relation of $G_{a_{\mathrm{u}}}$ is closed. In order to prove Theorem 1.2, we will not need to consider any Borel graphs other than $G_{a_{\mathbf{u}}}$. However, some of our lemmas are stated in the generality of any Borel graph of the form $G_{a}$, where $a$ is a free Borel action of $\mathbb{Z}^{d}$. If $G$ is a Borel graph on $X$, we let $[G]<\infty$ be the set of all finite subsets of $X$ that lie in a single connected component of $G$. This is a Borel subset of $[X]^{<\infty}$.

\section{Flows IN GRAPHS}

Our proof will use the following types of flows on graphs. Suppose $G$ is a locally finite graph with vertex set $V$, and $f: V \rightarrow \mathbb{R}$. An $f$-flow on $G$ is a real-valued function $\phi$ on the edges of $G$ such that $\phi(x, y)=-\phi(y, x)$ for every edge $(x, y)$ of $G$, and such that for every $x \in V$,

$$
f(x)=\sum_{y \in N(x)} \phi(x, y) .
$$

If $\epsilon>0$, then an $(\epsilon, f)$-flow is a real-valued function $\phi$ on the edges of $G$ such that $\phi(x, y)=-\phi(y, x)$ for every edge $(x, y)$ of $G$ and such that for every $x \in V$,

$$
\left|f(x)-\sum_{y \in N(x)} \phi(x, y)\right|<\epsilon .
$$

Suppose that $c$ is a nonnegative function on the edges of $G$ (where we may have $c(x, y) \neq c(y, x))$. We call $c$ a capacity function on $G$ and we say that an $f$-flow $\phi$ is bounded by $c$ if $\phi(x, y) \leq c(x, y)$ for every edge $(x, y)$ in $G$. We say that an $f$-flow $\phi$ is bounded if it is bounded by a constant capacity function.

If $\phi_{1}, \ldots, \phi_{n}$ are $f$-flows on a graph $G$, then their average $\frac{1}{n}\left(\sum_{i} \phi_{i}\right)$ is also an $f$-flow. The average of finitely many $(\epsilon, f)$-flows is similarly an $(\epsilon, f)$-flow. At a key step in our proof of Lemma 4.2, we will need to average over flows in this way. This is the reason why we have to use real-valued flows (instead of matchings) in our proof.

A folklore restatement of the max-flow min-cut theorem characterizes exactly when a finite graph $G$ admits an $f$-flow bounded by a capacity function $c$. Roughly, for every finite set $F$ of vertices, $\sum_{x \in F} f(x)$ should be at most the capacity of the edges leaving $F$.

Theorem 3.1. Suppose $G$ is finite graph on $X, f: X \rightarrow \mathbb{R}$ is a function, and $c$ is a capacity function for $G$. Then $G$ has an $f$-flow bounded by $c$ if and only if for every set $F \subseteq X$,

$$
\text { - } \sum_{\{(x, y) \in G: x \notin F \wedge y \in F\}} c(x, y) \leq \sum_{x \in F} f(x) \leq \sum_{\{(x, y) \in G: x \in F \wedge y \notin F\}} c(x, y) .
$$

Proof. The forward direction is clear, so we focus on the reverse. Define $G^{\prime}$ to be the finite graph containing $G$ as a subgraph where we add two vertices to $X$, a source $s$ and a sink $t$, and edges as follows. Add an edge $(s, x)$ to each $x \in X$ such that $f(x)>0$ and add an edge $(y, t)$ to each $y \in X$ such that $f(y)<0$. Define a capacity function $c^{\prime}$ for $G^{\prime}$ as follows. Let $c^{\prime}(x, y)=c(x, y)$ for every edge $(x, y)$ in 
$G$. For every edge $(s, x)$ incident to $s$, let $c^{\prime}(s, x)=f(x)$ and for every edge $(y, t)$ incident to $t$, let $c^{\prime}(y, t)=-f(y)$ and $c^{\prime}(t, y)=0$. Now apply the max-flow min-cut theorem [D] to the graph $G^{\prime}$ with source $s$, sink $t$, and capacity function $c^{\prime}$.

Take any $F \subseteq X$, and let $\{\{s\} \cup F,\{t\} \cup(X \backslash F)\}$ be a cut in $G^{\prime}$. One can show using either of the two inequalities on $f$ and $c$ that the capacity of this cut is greater than or equal to

$$
\sum_{\{x: f(x)>0\}} f(x)=-\sum_{\{x: f(x)<0\}} f(x) .
$$

which is the capacity of the cut containing just $s$ or just $t$. This value is therefore the minimum capacity of a cut in $G^{\prime}$, and the restriction to $G$ of the resulting maximal flow on $G^{\prime}$ is as required.

For finite graphs, there is no real difference in studying $f$-flows as defined above, and flows with a single source and single sink. This is because one can easily convert between these types of problems using the idea in the proof of Theorem 3.1 above. However, for infinite graphs, it becomes complicated to try and use a single source and sink to model an $f$-flow. (See for instance ABGPS]). For example, if $G$ is the infinite 3-regular tree and $f$ is the constant function 1 on the vertices of $G$, then $G$ admits an $f$-flow bounded by 1 . (Even though every vertex in the graph is a source, and there are no sinks).

In our proof, we will be constructing flows on infinite graphs. We remark that by taking ultralimits, one can characterize when a locally finite graph admits an $f$-flow bounded by a given capacity function. Note that in this case, we need both inequalities given in Theorem 3.1. We mention this result as a contrast to some of our results about Borel flows in infinite graphs. However, we will not use Theorem 3.2 in our proof of Theorem 1.2 .

Theorem 3.2 (Folklore). Suppose $G$ is locally finite graph on $X, f: X \rightarrow \mathbb{R}$ is a function, and $c$ is a capacity function such that $c(x, y)<\infty$ for every edge $(x, y)$ in $G$. Then $G$ has an $f$-flow bounded by $c$ if and only if for every finite set $F \subseteq X$,

$$
-\sum_{\{(x, y) \in G: x \notin F \wedge y \in F\}} c(x, y) \leq \sum_{x \in F} f(x) \leq \sum_{\{(x, y) \in G: x \in F \wedge y \notin F\}} c(x, y)
$$

Proof. By working in each connected component separately, we can assume that $G$ is connected. (This uses the axiom of choice). Fix a vertex $x$ in $G$ and for each $n \geq 0$ consider the graph $G_{n}$ which is the induced subgraph of $G$ on the vertex set $\left\{y: d_{G}(x, y) \leq n\right\}$. Let $G_{n}^{\prime}$ be the graph obtained by adding a single new vertex to $G_{n}$ and connecting it to each of the vertices $\left\{y: d_{G}(x, y)=n\right\}$ in $G_{n}$. Let $f_{n}$ be the function on the vertices of $G_{n}^{\prime}$ which is equal to $f$ on $\left\{y: d_{G}(x, y) \leq n\right\}$ and equal to $-\sum_{\left\{y: d_{G}(x, y) \leq n\right\}} f(y)$ at the new vertex. Clearly $G_{n}^{\prime}$ and $f_{n}$ satisfy the hypotheses of Theorem 3.1 for the capacity function $c^{\prime}$ which is equal to $c(x, y)$ on edges in $G_{n}$, and is infinite on the new edges in $G_{n}^{\prime}$. This is because the only sets $F$ yielding finite total capacities either lie in the interior of the $n$-ball around $x$, or their complement does.

Let $\phi_{n}$ be an $f_{n}$-flow for $G_{n}^{\prime}$. Let $U$ be a nonprincipal ultrafilter on $\mathbb{N}$. Define $\phi$ on the edges of $G$ by the ultralimit $\phi(e)=\lim _{U} \phi_{n}(e)$. The limit converges since $\left|\phi_{n}(e)\right| \leq c(e)$. It is straightforward to check that $\phi$ is an $f$-flow for $G$. 


\section{Constructing flows in $G_{a}$}

For this section, we fix a free action $a: \mathbb{Z}^{d} \curvearrowright X$ and a function $f: X \rightarrow \mathbb{R}$. Assuming $f$ satisfies the conditions given in Lemma 4.2, we give an explicit construction of an $f$-flow in the graph $G_{a}$. To prove Theorem 1.2, we will eventually apply Lemma 4.2 when the action $a$ is $a_{\mathbf{u}}$ for some $\mathbf{u}$ satisfying Lemma 2.1, and $f=\chi_{A}-\chi_{B}$, the difference between the characteristic functions of $A$ and $B$. Lemma 2.1 ensures that $f$ satisfies the hypothesis of Lemma 4.2. In this situation, the flow given by Lemma 4.2 will clearly be Borel (see Section 7 ).

For every $i>0$, let $\pi_{i}: \mathbb{Z}^{d} /\left(2^{i} \mathbb{Z}\right)^{d} \rightarrow \mathbb{Z}^{d} /\left(2^{i-1} \mathbb{Z}\right)^{d}$ be the canonical homomorphism. This yields the inverse limit

$$
\hat{\mathbb{Z}}^{d}=\varliminf_{i \geq 0} \mathbb{Z}^{d} /\left(2^{i} \mathbb{Z}\right)^{d}
$$

where elements of $\hat{\mathbb{Z}}^{d}$ are sequences $\left(h_{0}, h_{1}, \ldots\right)$ such that $\pi_{i}\left(h_{i}\right)=h_{i-1}$ for all $i>0$.

We begin by making several definitions which depend on $a$ and $f$. Below, if $F \subseteq X$ is finite, we will write $\sum_{F} f$ instead of $\sum_{x \in F} f(x)$.

Suppose $x \in X$ and $h \in \mathbb{Z}^{d} /\left(2^{n} \mathbb{Z}\right)^{d}$ for some $n>0$. View $h$ as a coset of $2^{n} \mathbb{Z}^{d}$ and recall that $R_{N}=\left\{\left(n_{1}, \ldots, n_{d}\right) \in \mathbb{Z}^{d}: 0 \leq n_{i}<N\right.$ for every $\left.i \leq d\right\}$. If we let

$$
P_{x, h}=\left\{\left(h^{\prime}+R_{2^{n}}\right) \cdot x: h^{\prime} \in h\right\},
$$

then $P_{x, h}$ is a partition of $[x]_{G_{a}}$ into sets of size $2^{n d}=\left|R_{2^{n}}\right|$. For every $y \in[x]_{G_{a}}$, let $[y]_{(x, h)}$ be the element of $P_{x, h}$ that contains $y$. Let

$$
Q_{x, h}(y)=\left\{[z]_{\left(x, \pi_{n}(h)\right)}: z \in[y]_{(x, h)}\right\},
$$

so $Q_{x, h}(y)$ is a partition of $[y]_{(x, h)}$ into $2^{d}$ many pieces.

Suppose $\gamma \in \mathbb{Z}^{d}$ is such that $|\gamma|_{\infty}=1$. Let $U_{x, h}(y, \gamma)$ be the set of $z \in[y]_{(x, h)}$ such that $\left(2^{n-1} \gamma\right) \cdot z \in[y]_{(x, h)}$ and there exists some $0 \leq i<2^{n-1}$ such that $y=(i \gamma) \cdot z$. Note that all $z \in U_{x, h}(y, \gamma)$ must come from a unique element of $Q_{x, h}(y)$, which we will call $Q_{x, h}(y, \gamma)$. Let $n_{x, h}(y, \gamma \cdot y)=\left|U_{x, y}(y, \gamma)\right|$.

Define a function $\phi_{x, h}$ on the edges of $G_{a}\left\lceil[x]_{G_{a}}\right.$ by

$$
\phi_{x, h}(y, \gamma \cdot y)=\frac{1}{2^{n d}} n_{x, h}(y, \gamma \cdot y) \sum_{Q_{x, h}(y, \gamma)} f .
$$

The rough idea is that $\phi_{x, h}$ is defined by working inside each element of $P_{x, h}$ and for each $z$ in this set, $\phi_{x, h}$ moves some mass at $z$ along a path of the form $z, \gamma \cdot z, \ldots,\left(2^{n-1} \gamma\right) \cdot z$ to the point $\left(2^{n-1} \gamma\right) \cdot z$. The definition of $\phi_{x, h}$ at an edge $(y, \gamma \cdot y)$ comes from summing the contribution of all the different $z$ 's whose associated path includes $(y, \gamma \cdot y)$.

Finally, for all $\left(h_{0}, h_{1}, \ldots\right) \in \hat{\mathbb{Z}}^{d}, y \in[x]_{G_{a}}$, and $\gamma \in \mathbb{Z}^{d}$ with $|\gamma|_{\infty}=1$, let

$$
\psi_{x,\left(h_{0}, \ldots, h_{n}\right)}(y, \gamma \cdot y)=\sum_{i=1}^{n}\left(\phi_{x, h_{i}}(y, \gamma \cdot y)-\phi_{x, h_{i}}(\gamma \cdot y, y)\right) .
$$

Our definition of $\psi_{x,\left(h_{0}, \ldots, h_{n}\right)}$ is chosen so that the "error" at $y$ which prevents $\psi_{x,\left(h_{0}, \ldots, h_{n}\right)}$ from being a flow is exactly the average value of $f$ over $[y]_{\left(x, h_{n}\right)}$. This is the content of Lemma 4.1 
Lemma 4.1. For every $x \in X,\left(h_{0}, h_{1} \ldots\right) \in \hat{\mathbb{Z}}^{d}, n>0$ and $y \in[x]_{G_{a}}$,

$$
f(y)-\sum_{|\gamma|_{\infty}=1} \psi_{x,\left(h_{0}, \ldots, h_{n}\right)}(y, \gamma \cdot y)=\frac{1}{2^{n d}} \sum_{[y]_{\left(x, h_{n}\right)}} f
$$

Proof. We work by induction. For the base case of $n=0$, the left hand size is $f(y)$ since the summation defining $\psi_{x,\left(h_{0}\right)}$ is empty. The right hand side is also $f(y)$ since $[y]_{\left(x, h_{0}\right)}=\{y\}$.

Let the left hand size of the above equation be

$$
\theta_{n}(y)=f(y)-\sum_{|\gamma|_{\infty}=1} \psi_{x,\left(h_{0}, \ldots, h_{n}\right)}(y, \gamma \cdot y) .
$$

Note that

$$
\theta_{n}(y)=\theta_{n-1}(y)-\left(\sum_{|\gamma|_{\infty}=1} \phi_{x, h_{n}}(y, \gamma \cdot y)-\phi_{x, h_{n}}(-\gamma \cdot y, y)\right)
$$

where we have changed $\phi_{x, h_{n}}(\gamma \cdot y, y)$ to $\phi_{x, h_{n}}(-\gamma \cdot y, y)$ in the second term in the summation, by using the fact that we are summing over all $\gamma$ with $|\gamma|_{\infty}=1$. Note that $(-\gamma \cdot y, y)=(-\gamma \cdot y, \gamma \cdot(-\gamma) \cdot y)$ is an edge oriented in the "direction" of $\gamma$.

We now compute this sum. Fix $z \in U_{x, h_{n}}(y, \gamma)$ so that $z \in[y]_{\left(x, h_{n}\right)}, 2^{n-1} \gamma \cdot z \in$ $[y]_{\left(x, h_{n}\right)}$ and $y=(i \gamma) \cdot z$ for some $0 \leq i<2^{n-1}$. If $y \neq z$, then $z$ is counted in both $n_{x, h_{n}}(y, \gamma \cdot y)$ and $n_{x, h_{n}}(-\gamma \cdot y, y)$. Moreover it contributes the same amount to $\phi_{x, h_{n}}(y, \gamma \cdot y)$ and $\phi_{x, h_{n}}(-\gamma \cdot y, y)$. Using this fact to cancel corresponding terms and summing over $\gamma$, we get

$$
\begin{aligned}
\sum_{|\gamma|_{\infty}=1} \phi_{h_{n}, x}(y, \gamma \cdot y) & -\phi_{h_{n}, x}(-\gamma \cdot y, y) \\
& =\frac{1}{2^{n d}}\left(\left|S_{y}\right| \sum_{[y]_{\left(x, h_{n}-1\right)}} f-\sum_{z \in\left\{\left(2^{n-1} \gamma\right) \cdot y: \gamma \in S_{y}\right\}} \sum_{[z]_{\left(x, h_{n-1}\right)}} f\right) .
\end{aligned}
$$

where $S_{y}=\left\{\gamma:|\gamma|_{\infty}=1 \wedge\left(2^{n-1} \gamma\right) \cdot y \in[y]_{\left(x, h_{n}\right)}\right\}$. Note that $\left\{\left[\left(2^{n-1} \gamma\right) \cdot y\right]_{\left(x, h_{n-1}\right)}\right.$ : $\left.\gamma \in\left(S_{y} \cup\{0\}\right)\right\}=Q_{x, h_{n}}(y)$ and so $S_{y}$ has $2^{d}-1$ elements since $Q_{x, h}(y)$ has $2^{d}$ many elements.

Using our induction hypothesis that $\theta_{n-1}(y)=\frac{1}{2^{(n-1) d}} \sum_{[y]_{\left(x, h_{n-1}\right)}} f$ and simplifying, we get:

$$
\theta_{n}(y)=\frac{1}{2^{n d}}\left(\sum_{[y]_{\left(x, h_{n}-1\right)}} f+\sum_{z \in\left\{\left(2^{n-1} \gamma\right) \cdot y: \gamma \in S_{y}\right\}} \sum_{[z]]_{\left(x, h_{n-1}\right)}} f\right)
$$

which is equal to $\frac{1}{2^{n d}} \sum_{[y]_{\left(x, h_{n}\right)}} f$ using the fact from above that $\left\{\left[\left(2^{n-1} \gamma\right) \cdot y\right]_{\left(x, h_{n-1}\right)}\right.$ : $\left.\gamma \in\left(S_{y} \cup\{0\}\right)\right\}=Q_{x, h_{n}}(y)$ is a partition of $[y]_{\left(x, h_{n}\right)}$.

Lemma 4.1 implies that for every $x \in X$ and $\left(h_{0}, h_{1} \ldots\right) \in \hat{\mathbb{Z}}^{d}$, the function $\lim _{n \rightarrow \infty} \psi_{x,\left(h_{0}, \ldots, h_{n}\right)}(y, z)$ will be an $f$-flow provided it converges everywhere and $\lim _{n \rightarrow \infty} \frac{1}{2^{n d}} \sum_{[y]_{\left(x, h_{n}\right)}} f \rightarrow 0$ everywhere. However, if $a: \mathbb{Z}^{d} \curvearrowright X$ is a Borel action, then we cannot hope to pick a single point $x$ out of each connected component of $G_{a}$ in a Borel way to use as a base point in this construction. (For example, for the action $a_{\mathbf{u}}$, a set which meets $\mathbb{T}^{k}$ exactly once in each orbit must be nonmeasurable). 
Instead, we will average the above construction over every possible element of $\hat{\mathbb{Z}}^{d}$, and then use the fact that the resulting function does not depend on the base point $x$ that we choose.

For every $x \in X$, define

$$
\psi_{x}(y, z)=\sum_{n>0} \frac{1}{2^{n d}} \sum_{h \in \mathbb{Z}^{d} /\left(2^{n} \mathbb{Z}\right)^{d}} \phi_{x, h}(y, z)-\phi_{x, h}(z, y) .
$$

Finally, let $\psi$ be defined on every edge $(y, z)$ in $G_{a}$ by

$$
\psi(y, z)=\psi_{y}(y, z)
$$

Lemma 4.2. Suppose there is a function $\Phi: \mathbb{N} \rightarrow \mathbb{R}$ such that for every $y \in X$

$$
\left|\sum_{R_{2^{n}} \cdot y} f\right|<\Phi\left(2^{n}\right)
$$

and

$$
c=\frac{1}{2^{d-1}} \sum_{n=0}^{\infty} \frac{\Phi\left(2^{n}\right)}{2^{n(d-1)}}
$$

is finite. Then $\psi$ is an $f$-flow bounded by $c$.

Proof. We begin by showing that for every $x \in X, \psi_{x}$ is an $f$-flow of $G \uparrow[x]_{G_{a}}$ bounded by $c$.

If $h_{n} \in \mathbb{Z}^{d} /\left(2^{n} \mathbb{Z}\right)^{d}$, then since $n_{x, h_{n}}(y, s) \leq 2^{n-1}$, and $\left.\mid \sum_{Q_{x, h}(y, \gamma)} f\right) \mid \leq \Phi\left(2^{n-1}\right)$, we get $\left|\phi_{x, h}(y, \gamma \cdot y)\right| \leq 2^{n-1} \frac{\Phi\left(2^{n-1}\right)}{2^{n d}}$ Hence,

$$
\left|\psi_{x}(y, z)\right| \leq \sum_{n=1}^{\infty} 2 \cdot 2^{n-1} \frac{\Phi\left(2^{n-1}\right)}{2^{n d}}=\frac{1}{2^{d-1}} \sum_{n=1}^{\infty} \frac{\Phi\left(2^{n-1}\right)}{2^{(n-1)(d-1)}}=c .
$$

If we consider the first $n$ terms in the summation defining $\psi_{x}$, this is equal to the average of $\psi_{x,\left(h_{0}, \ldots, h_{n}\right)}$ over all sequences $\left(h_{0}, \ldots, h_{n}\right)$ with $h_{i} \in \mathbb{Z}^{d} /\left(2^{i} \mathbb{Z}\right)^{d}$ and $\pi_{i}\left(h_{i}\right)=h_{i-1}$. This is because there are the same number of these sequences $\left(h_{0}, \ldots, h_{n}\right)$ containing any given $h_{i} \in \mathbb{Z}^{d} /\left(2^{i} \mathbb{Z}\right)^{d}$. Each such $\psi_{x,\left(h_{0}, \ldots, h_{n}\right)}$ is an $(\epsilon, f)$-flow for $\epsilon=\Phi\left(2^{n}\right) / 2^{n d}$ by Lemma 4.1. Since the average of finitely many $(\epsilon, f)$-flows is an $(\epsilon, f)$-flow, it follows that $\psi_{x}$ is a limit of $(\epsilon, f)$-flows with $\epsilon=$ $\Phi\left(2^{n}\right) / 2^{n d}$, which approaches 0 as $n$ goes to $\infty$. Finally, $\psi_{x}(y, z)=-\psi_{x}(z, y)$ by definition. This finishes the proof that $\psi_{x}$ is an $f$-flow bounded by $c$.

To show that $\psi$ is an $f$-flow of $G_{a}$, it is enough to show that for all $x \in X$ and $g \in \mathbb{Z}^{d}, \psi_{x}=\psi_{g \cdot x}$. Now $P_{g \cdot x,-g+h}=P_{x, h}$ and so $\phi_{g \cdot x,-g+h}=\phi_{x, h}$. From this we can conclude that $\psi_{g \cdot x}=\psi_{x}$, since each term in the summation defining $\psi_{x}$ averages $\phi_{x, h}$ over all $h \in \mathbb{Z}^{d} /\left(2^{n} \mathbb{Z}\right)^{d}$, which is equal to the average of $\phi_{g \cdot x,-g+h}$ over all $h \in \mathbb{Z}^{d} /\left(2^{n} \mathbb{Z}\right)^{d}$, and hence the average of $\phi_{g \cdot x, h}$ over all $h \in \mathbb{Z}^{d} /\left(2^{n} \mathbb{Z}\right)^{d}$.

\section{INTEGRAL Borel FlOWS}

Suppose $G$ is a graph on $X$, and $f: X \rightarrow \mathbb{Z}$ is a function. An integral $f$-flow is an $f$-flow $\phi$ so that $\phi(x, y)$ is an integer for every edge $(x, y)$ in $G$. In this section, we consider the problem of turning real-valued $f$-flows into integral $f$-flows.

Classically, the question of when a locally finite graph admits an integral $f$-flow is easy to answer. It is usually called the integral flow theorem. 
Theorem 5.1 (The integral flow theorem [D]). Suppose $G$ is a locally finite graph on $X, c$ is a capacity function for $G$ that takes integer values, and $f: X \rightarrow \mathbb{Z}$ also takes integer values. If there is an $f$-flow bounded by $c$, then there is an integral $f$-flow bounded by $c$.

Proof. Suppose first that $G$ is a finite graph. Consider the graph $G^{\prime}$ defined in the proof of Theorem [3.1. If we use the Ford-Fulkerson algorithm (see [D]) to find a maximal flow for $G^{\prime}$, it will make a flow with only integer values.

For infinite graphs, the theorem follows from the finite case using the same idea as the proof of Theorem 3.2. This is because an ultralimit of integer valued functions is integer valued.

We also have the following folklore theorem which shows that if $f$ takes integer values, then we can find an integral $f$-flow "close" to any real-valued $f$-flow.

Corollary 5.2. Suppose $G$ is locally finite graph on $X$ and $f: X \rightarrow \mathbb{Z}$ takes integer values. If $\phi$ is an $f$-flow, then there is an integral $f$-flow $\psi$ such that

$$
|\phi(x, y)-\psi(x, y)|<1
$$

for every edge $(x, y)$ in $G$.

Proof. Let

$$
\phi^{\prime}(x, y)= \begin{cases}\lfloor\phi(x, y)\rfloor & \text { if } \phi(x, y) \geq 0 \\ \lceil\phi(x, y)\rceil & \text { if } \phi(x, y)<0\end{cases}
$$

Then $\phi^{\prime}(x, y)=-\phi^{\prime}(x, y)$, and $\phi^{\prime}$ is an integral $f^{\prime}$-flow for the function $f^{\prime}(x)=$ $\sum_{y \in N(x)} \phi^{\prime}(x, y)$. Thus, $\left(\phi-\phi^{\prime}\right)$ is an $\left(f-f^{\prime}\right)$-flow which is bounded by the capacity function where $c(x, y)=0$ if $\phi(x, y)-\phi^{\prime}(x, y) \leq 0$ and $c(x, y)=1$ otherwise. Now applying Theorem 5.1, there is an integral $\left(f-f^{\prime}\right)$-flow $\phi^{\prime \prime}$ which is bounded by $c$. Finally, adding again we see that $\psi=\phi^{\prime \prime}+\phi^{\prime}$ is an integral $f$-flow, and $|\phi(x, y)-\psi(x, y)|<1$.

Note that since $|\phi(x, y)-\psi(x, y)|<1$ in Corollary 5.2. if $\phi(x, y)$ is already an integer, then $\psi(x, y)=\phi(x, y)$.

Now in the Borel setting, we have the following corollary of [L88] which implies that we cannot always turn a real valued Borel $f$-flow into an integral Borel $f$-flow.

Corollary 5.3 ([L88). There is a 2-regular acyclic Borel graph $G$ on $X$ and a Borel function $f: X \rightarrow \mathbb{Z}$ such that $G$ has a Borel $f$-flow, but does not have an integral Borel $f$-flow.

Proof. Let $G$ be the graph defined by Laczkovich in [L88. This graph is a 2-regular acyclic Borel graph $G$ on a standard Borel space $X$, that admits a bipartition into two Borel sets $X_{0}$ and $X_{1}$. By L88 this graph has no Borel (or even Lebesguemeasurable) perfect matching. Now let $f: X \rightarrow\{-1,1\}$ be the function where $f(x)=1$ if $x \in X_{0}$ and $f(x)=-1$ if $x \in X_{1}$. If we let $\phi(x, y)=1 / 2$ and $\phi(y, x)=-1 / 2$ for every edge $(x, y)$ in $G$ where $x \in X_{0}$ and $y \in X_{1}$, then $\phi$ is clearly a Borel $f$-flow.

However, $G$ does not have an integer-valued Borel $f$-flow. For a contradiction, suppose $\psi$ was in integer-valued Borel $f$-flow for $G$. Then the set of edges $(x, y)$ such that $x \in X_{0}$ and $y \in X_{1}$ and $\psi(x, y)>0$ would be a Borel perfect matching of $G$. 
Despite this, we do have the following "Borel integral flow theorem" for graphs induced by free Borel actions of $\mathbb{Z}^{d}$ for $d \geq 2$.

Lemma 5.4. Suppose $d \geq 2, a: \mathbb{Z}^{d} \curvearrowright X$ is a free Borel action, and $G_{a}$ is the associated graph. Then if $f: X \rightarrow \mathbb{Z}$ is a Borel function and $\phi$ is a Borel $f$-flow for $G$, then there is an integral Borel $f$-flow $\psi$ such that $|\phi-\psi| \leq 3^{d}$.

In our proof of Theorem 1.2 we will use Lemma 5.4 to turn the real-valued Borel flow constructed in Lemma 4.2 into an integral Borel flow.

Our proof of Lemma 5.4 uses the following result of Gao, Jackson, Krohne, and Seward (see Appendix A). Their theorem answers a question originally due to Ben Miller.

Suppose $F$ is a finite set of vertices in a graph $G$, let $\partial F$ be the set of edges that are incident on one vertex in $F$ and one vertex not in $F$. Now let $\partial^{1} F=\partial F$, and let $\partial^{n+1} F$ be the set of edges that are in $\partial^{n} F$ or share a vertex with an edge in $\partial^{n} F$. Finally, let $\partial_{\text {vis }(\infty)} F \subseteq \partial F$ be the set of edges in the boundary of $F$ that are "visible from infinity". That is, $\partial_{\text {vis }(\infty)} F$ is the set of edges $e \in \partial F$ such that the unique $x \notin F$ incident to $e$ is such that the connected component of $x$ in $G-\partial F$ is infinite.

Theorem 5.5 (Gao, Jackson, Krohne, and Seward). Suppose $d \geq 1, n>0$, and $a: \mathbb{Z}^{d} \curvearrowright X$ is a free Borel action of $\mathbb{Z}^{d}$ on a standard Borel space $X$, and $G_{a}$ is the associated graph. Then there is a Borel set $C \subseteq[X]^{<\infty}$ such $\bigcup C=X$, for every distinct $R, S \in C, \partial^{n} R$ and $\partial^{n} S$ are disjoint, and every $S \in C$ is connected and has $\partial S=\partial_{v i s(\infty)} S$.

For an annoucement of this theorem, see the paragraph following Corollary 1.8 in GJKS.

The point of this theorem is that the elements of $C$ cover the whole space $X$, and their boundaries can be chosen to be arbitrarily far apart. The condition that $\partial S=\partial_{\text {vis }(\infty)} S$ is incidental and is included just to make Lemma 5.6] a little simpler. Given any connected $S \subseteq X$, let $\tilde{S}$ be the set of vertices in $S$ together with all $x \in X$ that are in some finite connected component of $G-\partial S$. The idea here is that $\tilde{S}$ is obtained by filling in any "holes" inside $S$. It is clear that $\partial \tilde{S} \subseteq \partial S$, and that $\partial_{\text {vis }(\infty)} \tilde{S}=\partial \tilde{S}$. Hence, if $C \subseteq[G]^{<\infty}$ satisfies all the conditions of the theorem except the condition on $\partial_{\text {vis }(\infty)}$, then we can simply replace $C$ with $\{\tilde{S}: S \in C\}$ to satisfy this last condition.

We need a short combinatorial lemma. Recall than an Euler cycle in a finite graph is a closed walk that includes each edge in the graph exactly once.

Lemma 5.6. Suppose $d \geq 2, a: \mathbb{Z}^{d} \curvearrowright X$ is a free action, $G_{a}$ is the associated graph, and $F \subseteq X$ is a finite $G_{a}$-connected set with $\partial_{\text {vis }(\infty)} F=\partial F$. Let $H_{\partial F}$ be the graph whose vertex set is the unordered edges in $\partial F$, that is, $\{\{x, y\}:(x, y) \in \partial F\}$ and where distinct $\{x, y\},\{z, w\}$ are adjacent in $H_{\partial F}$ if there is a 3-cycle in $G_{a}$ that includes them both. Then $H_{\partial F}$ has an Euler cycle.

Proof. By Euler's theorem, we need to show that $H_{\partial F}$ is connected and every vertex of $H_{\partial F}$ has even degree.

We begin by showing every edge has even degree. Fix $(x, y) \in \partial F$. Any 3-cycle in $G_{a}$ that contains $(x, y)$ must contain exactly one other edge in $\partial F$. Thus, it suffices to show that there are an even number of 3-cycles in $G_{a}$ containing the edge $(x, y)$. Let $(x, y)=(x, \gamma \cdot x)$ where $|\gamma|_{\infty}=1$. Then the number of 3-cycles 
containing $(x, y)$ is equal to the number of $\delta$ with $|\delta|_{\infty}=1$ and $|\gamma-\delta|_{\infty}=1$. If $\gamma=\left(\gamma_{1}, \ldots, \gamma_{d}\right)$, then this $\delta=\left(\delta_{1}, \ldots, \delta_{d}\right)$ must have $\delta_{i} \in\{-1,0,1\}$ if $\gamma_{i}=0$, $\delta_{i} \in\{0,1\}$ if $\gamma_{i}=1$, and $\delta_{i} \in\{-1,0\}$ if $\gamma_{i}=-1$. Thus, if there are $k$ many values of $i$ such that $\gamma_{i}=0$, then the number of $\delta$ with this property is $3^{k} 2^{d-k}-2$, where we subtract 2 since neither $\delta$ nor $\gamma-\delta$ can be equal to 0 . To finish, note that $3^{k} 2^{d-k}-2$ is even since $k<d$.

Next, we claim that $H_{\partial F}$ is connected. To see this will use Ti. First, we claim that the set of all 3-cycles in $G_{a}$ generates the cycle space of $G_{a}$. That is, every cycle in $G_{a}$ is a sum of finitely many 3 -cycles, where we add edges in the cycles modulo 2 (see [Ti]). This is easy to see, and we sketch an argument in the case where $a$ is the translation action of $\mathbb{Z}^{d}$ on itself. Let $e_{i}$ be the $i$ th element of the usual basis for $\mathbb{Z}^{d}$. Let $T$ be the spanning subtree of $G_{a}$ where for each $x=\left(x_{1}, x_{2}, \ldots, x_{d}\right) \in \mathbb{Z}^{d}$, we have $\left(x, e_{i} \cdot x\right) \in T$ if $x_{j}=0$ for all $j>i$. Given an edge $(x, y) \in G_{a}$ such that $(x, y) \notin T$, let $C_{(x, y)}$ be the unique cycle created by adding $(x, y)$ to $T$. An easy induction shows that every such $C_{(x, y)}$ is a sum of 3-cycles, but clearly any cycle in $G_{a}$ is a sum of cycles of the form $C_{(x, y)}$.

Since $F$ is connected and $\partial_{\mathrm{vis}(\infty)} F=\partial F$, if we choose any $x \in F$ and $y \notin F$ where $y \in[x]_{G_{a}}$, then $\partial F$ is a minimal set of edges separating $x$ and $y$ in the sense that any proper subset of $\partial F$ does not separate $x$ and $y$. Hence, by [Ti, Lemma 1], if $H_{\partial F}$ had two connected components $\Pi_{1}$ and $\Pi_{2}$, then there would be some 3 -cycle in $G_{a}$ that intersects both $\Pi_{1}$ and $\Pi_{2}$. But this is clearly a contradiction.

We are now ready to prove Lemma 5.4 .

Proof of Lemma 5.4. Let $\phi$ be a Borel $f$-flow for $G_{a}$. Let $C \subseteq X^{\infty}$ be as in Theorem [5.5. with $n=3$. We use $n=3$ here for the following reason: if $R, S \in C$ and we change $\phi$ separately on $\partial^{2} R$ and $\partial^{2} S$ in a such a way that it remains a flow after each individual modification, then if we combine both modifications the result will also be a flow.

We define another Borel $f$-flow $\phi^{\prime}$ as follows. Let $\phi^{\prime}(x, y)=\phi(x, y)$ if $(x, y)$ is not in $\partial^{2} F$ for any $F \in C$. If $(x, y) \in \partial^{2} F$ for some $F \in C$, then there is a unique such $F$. Let $\left(\left(e_{1}, e_{1}^{\prime}, e_{1}^{\prime \prime}\right), \ldots,\left(e_{n}, e_{n}^{\prime}, e_{n}^{\prime \prime}\right)\right)$ be the sequence of 3 -cycles in $G_{a}$ associated to the lexicographically least Euler cycle of $H_{\partial F}$, where we are representing each 3 -cycle by the edges contained in it. An Euler cycle of $H_{\partial F}$ exists by Lemma 5.6. We may arrange these 3 -cycles so that for all $i$, we have $e_{i}, e_{i}^{\prime} \in \partial F, e_{i}^{\prime \prime} \notin \partial F$, and $e_{i}^{\prime}=e_{i+1}$. Orient the edges so that $\left(e_{i}, e_{i}^{\prime}, e_{i}^{\prime \prime}\right)=\left(\left(x_{i}, y_{i}\right),\left(y_{i}, z_{i}\right),\left(z_{i}, x_{i}\right)\right)$.

Let $\phi_{0}^{F}=\phi$. Given $\left(e_{i}, e_{i}^{\prime}, e_{i}^{\prime \prime}\right)$, let $\alpha_{i}=\phi_{i}^{F}\left(e_{i}\right)-\left\lfloor\phi_{i}^{F}\left(e_{i}\right)\right\rfloor$. Then define $\phi_{i+1}^{F}(u, v)=\phi_{i}^{F}(u, v)$ if $(u, v)$ is not an edge in the cycle $\left(e_{i}, e_{i}^{\prime}, e_{i}^{\prime \prime}\right)$, and otherwise let $\phi_{i+1}^{F}(u, v)=\phi_{i}^{F}(u, v)-\alpha_{i}$ if $(u, v)$ is oriented the same direction as the cycle $e_{i}, e_{i}^{\prime}, e_{i}^{\prime \prime}$, and $\phi_{i+1}^{F}(u, v)=\phi_{i}^{F}(u, v)+\alpha_{i}$ if $(u, v)$ is oriented in the opposite direction. Hence, $\phi_{i+1}^{F}$ is still an $f$-flow since we are modifying $\psi_{i}^{F}$ only by adding the same amount to each edge going around a single cycle. Finally, let $\phi^{\prime}(x, y)=\phi_{n}^{F}(x, y)$.

We claim that if $e \in \partial F$, then $\phi^{\prime}(e)$ will be an integer. First suppose that $e \neq e_{n}^{\prime}$. Then if $j$ is the largest number such that $e=e_{j}$ (up to direction), we define $\phi_{j}^{F}(e)$ to be an integer, and $e$ cannot equal $e_{k}^{\prime}$ for any $k>i$ since $e_{k}^{\prime}=e_{k+1}$, hence $\phi_{k}^{F}(e)=\phi_{j}^{F}(e)$ for all $k>i$. Thus $\phi^{\prime}(e)$ is an integer. If $e=e_{n}^{\prime}$, then since $f$ is integer valued, the total flow out of $F$ must be an integer. So since $\phi^{\prime}$ takes integer values on all the other edges in $\partial F, \phi^{\prime}(e)$ must also be an integer. 
Note that $\left|\phi^{\prime}(x, y)-\phi(x, y)\right| \leq 3^{d}-1$ for every edge $(x, y)$. This is because every node in $H_{\partial F}$ has degree at most $3^{d}-1$, and we change the flow on each edge by at most 1 as we go around the Euler cycle.

Now let $D$ be the set of edges $(x, y)$ in $G_{a}$ such that $(x, y) \in \partial F$ for some $F \in C$. $G_{a}-D$ has finite connected components, since $\bigcup C=X$. Let $K$ be a connected component of $G_{a}-D$. If $\theta$ is an integer-valued function defined on the edges of $K$, let $\phi_{\theta}^{\prime}(x, y)=\theta(x, y)$ if the edge $(x, y)$ is in $K$, and $\phi^{\prime}(x, y)$ otherwise. By Corollary [5.2, there is a integer valued function $\theta$ defined on the edges of $K$ so that $\phi_{\theta}^{\prime}(x, y)$ is an $f$-flow, and $\left|\phi_{\theta}^{\prime}-\phi^{\prime}\right|<1$.

Let $\psi$ be the Borel $f$-flow on $G_{a}$ defined as follows. Let $\psi(x, y)=\phi^{\prime}(x, y)$ if $(x, y) \in D$. If $(x, y) \notin D$, then let $K$ be the connected component of $G_{a}-D$ containing $(x, y)$, let $\theta$ be the lexicographically least integer-valued function on the edges of $K$ such that $\phi_{\theta}^{\prime}$ is an $f$-flow where $\left|\phi_{\theta}^{\prime}-\phi^{\prime}\right|<1$, and let $\psi(x, y)=$ $\phi_{\theta}^{\prime}(x, y)$.

\section{Proof of Theorem 1.2}

In order to prove Theorem 1.2 using the fewest number of pieces in our equidecomposition, we use the following lemma due to Gao and Jackson, which was an important ingredient in their proof that Borel actions of abelian groups are hyperfinite. In Remark 6.2 we describe how one can prove Theorem 1.2 without using this black box.

Lemma 6.1 (GJ]). Suppose $a: \mathbb{Z}^{d} \curvearrowright X$ is a free Borel action of $\mathbb{Z}^{d}$ on a standard Borel space $X$ and $n>0$. Then there is a Borel set $C \subseteq[X]^{<\infty}$ such that $C$ partitions $X$ and every $S \in C$ is a set of the form $\left\{\left(n_{1}, \ldots, n_{d}\right) \cdot x: 0 \leq n_{i}<N_{i}\right\}$ for some $x \in X$ and sequence $N_{1}, \ldots, N_{d}$ where $N_{i}=n$ or $N_{i}=n+1$.

Roughly, the above lemma states that there is a Borel tiling of the action $a$ using rectangles each of whose side lengths is $n$ or $n+1$.

Proof of Theorem 1.2. As discussed at the beginning of Section 2 we may assume that $A, B \subseteq \mathbb{T}^{k}$. Let $d \geq 2$ be such that $d>2 k /(k-\Delta(\partial A))$ and pick $\mathbf{u} \in\left(T^{k}\right)^{d}$ such that the action $a_{\mathbf{u}}$ is free and satisfies Lemma 2.1 for both sets $A$ and $B$. Hence, there is some $M$ and $\epsilon>0$ such that

$$
D\left(F_{N}\left(x, a_{\mathbf{u}}\right), A\right) \leq M N^{-1-\epsilon} \quad \text { and } \quad D\left(F_{N}\left(x, a_{\mathbf{u}}\right), B\right) \leq M N^{-1-\epsilon}
$$

for every $x \in \mathbb{T}^{k}$ and $N>0$.

Now consider the graph $G_{a_{\mathbf{u}}}$ and the function $f=\chi_{A}-\chi_{B}$, the difference between the characteristic functions of $A$ and $B$. Since $\lambda(A)=\lambda(B)$, by the definition of discrepancy and $f$, for every $x \in \mathbb{T}^{k}$ and $n>0$,

$$
\left|\sum_{F_{2^{n}}\left(x, a_{\mathbf{u}}\right)} f\right|=2^{n d}\left|D\left(F_{2^{n}}\left(x, a_{\mathbf{u}}\right), A\right)-D\left(F_{2^{n}}\left(x, a_{\mathbf{u}}\right), B\right)\right| \leq 2 M 2^{n(d-1-\epsilon)} .
$$

Thus, by Lemma 4.2, letting $\Phi\left(2^{n}\right)=M 2^{n(d-1-\epsilon)+1}$, there is a bounded Borel $f$-flow for the graph $G_{a_{\mathrm{u}}}$. By Lemma 5.4, there is a bounded integral Borel $f$-flow for the graph $G_{a_{\mathrm{u}}}$. Call this integral Borel flow $\psi$, and suppose that $\psi$ is bounded by the constant $c$.

For each $n$, by Lemma 6.1 let $C_{n} \subseteq\left(\mathbb{T}^{k}\right)^{<\infty}$ be a Borel tiling of the action $a_{\mathbf{u}}$ by rectangles of side lengths $n$ or $n+1$. For each $x \in \mathbb{T}^{k}$, let $V_{n}(x)$ be the unique 
element of $C_{n}$ that contains $x$. Now for every $x \in \mathbb{T}^{k},\left|\partial V_{n}(x)\right| \leq 2 d \cdot 3^{d} \cdot(n+1)^{d-1}$ which is $O\left(n^{d-1}\right)$. Next, since there is some $x^{\prime} \in V_{n}(x)$ such that $F_{n}\left(x^{\prime}, a_{\mathbf{u}}\right) \subseteq$ $V_{n}(x)$, we have that $\left|A \cap V_{n}(x)\right| \geq \lambda(A) n^{d}-M n^{d-1-\epsilon}$. Hence, there is some $K$ so that

$$
c\left|\partial V_{K}(x)\right| \leq\left|A \cap V_{K}(x)\right| \quad \text { and } \quad c\left|\partial V_{K}(x)\right| \leq\left|B \cap V_{K}(x)\right|
$$

for every $x \in \mathbb{T}^{k}$. Fix this $K$ and let $C=C_{K}$.

For each $R \in C$, let $N(R)$ be the set of $S \in C$ such that $S \neq R$ and $\partial S \cap \partial R \neq \emptyset$. Note that $N(R)$ is finite. Given $S \in N(R)$, let

$$
\Psi(R, S)=\sum_{\{(x, y): x \in R \wedge y \in S\}} \psi(x, y)
$$

so $\Psi(R, S)$ is integer-valued, $\Psi(R, S)=-\Psi(S, R)$ and

$$
\sum_{S \in N(R)} \Psi(R, S)=|R \cap A|-|R \cap B| .
$$

Note also that $\sum_{S \in N(R)}|\Psi(R, S)|$ is less than $|R \cap A|$ and $|R \cap B|$ by our choice of $K$ and if $S \in N(R)$, then for any $x \in R$ and $y \in S$, there is a $\gamma \in \mathbb{Z}^{d}$ with $|\gamma|_{\infty}<2 K+4$ such that $\gamma \cdot x=y$.

Essentially, if we let $G_{C}$ be the graph with vertex set $C$ where $R$ is adjacent to $S$ if $\partial R \cap \partial S$, then $\Psi$ is a flow on this graph for the function $f(R)=\sum_{R} \chi_{A}-\chi_{B}$, and $N$ is the neighborhood relation on this graph.

To show that $A$ and $B$ are $a_{\mathbf{u}}$-equidecomposable using Borel pieces, it suffices to construct a Borel bijection $g: A \rightarrow B$ such that for all $x \in A, g(x)=\gamma \cdot x$ for some $\gamma$ such that $|\gamma|_{\infty}<2 K+4$. Then the pieces in our equidecomposition will be $\{x \in A: g(a)=\gamma \cdot x\}$ for each $\gamma$ with $|\gamma|_{\infty}<2 K+4$.

Our idea for constructing $g$ is that for every $R, S \in C$, if $\Psi(R, S)>0$ we should map $\Psi(R, S)$ many points from $A \cap R$ to point of $B \cap S$. After doing this for all pairs $R, S$, there will be an equal number of points of $A$ and $B$ left in each $R \in C$, so we map the remaining points in $A \cap R$ to the points of $B \cap R$.

Fix a Borel linear ordering $<_{C}$ of $C$, and a Borel linear ordering $<$ of $\mathbb{T}^{k}$. For each $R \in C$, inductively let $A(R, S) \subseteq A \cap R$ be the least $\Psi(R, S)$ many elements of $A \cap R$ that are not in $A\left(R, S^{\prime}\right)$ for any $S^{\prime} \in N(R)$ where $S^{\prime}<_{C} S$. Similarly, for each $R \in C$, let $B(R, S) \subseteq B \cap R$ be the first $\Psi(R, S)$ many elements of $B \cap R$ that are not in $B\left(R, S^{\prime}\right)$ for any $S^{\prime} \in N(R)$ with $S^{\prime}<_{C} S$. Finally, let $A^{\prime}(R)=$ $\left.A \cap R \backslash \bigcup_{\{S \in N(R): \Psi(R, S)>0\}} A(R, S)\right\}$ and $B^{\prime}(R)=B \cap R \backslash \bigcup_{\{S \in N(R): \Psi(R, S)\}} B(R, S)$. By the properties of $\Psi$ listed above, $\left|A^{\prime}(R)\right|=\left|B^{\prime}(R)\right|$ for every $R \in C$. Define $g: A \rightarrow B$ as follows. Given $x \in A$, let $R$ be the unique element of $C$ containing $x$. If there is some $S \in N(R)$ such that $x \in A(R, S)$, and $x$ is the lth-least element of $A(R, S)$, then let $g(x)$ be the $l$ th-least element of $B(S, R)$. If not, then $x \in A^{\prime}(R)$. If $x$ is the lth-least element of $A^{\prime}(R)$, then let $g(x)$ be the $l$ th-least element of $B^{\prime}(R)$.

Remark 6.2. We sketch an alternate proof of Theorem 1.2 without using Lemma 6.1 By Theorem A.1. for each $n$, there is a Borel maximal $n$-discrete set $C_{n}$ for $G_{a_{\mathbf{u}}}$. Note that the $n / 2$-balls around points in $C_{n}$ are pairwise disjoint. Given $x \in C_{n}$, let $V_{n}(x)$ be the Voronoi cell determined by the seed $x$ in the graph $G_{a_{\mathbf{u}}}$. That is, $V_{n}(x)$ is the set of $y \in \mathbb{T}^{k}$ such that $x$ is the <-least element of $V_{n}$ such that $d(y, x) \leq d(y, z)$ for all $z \in \mathbb{T}^{k}$. Note that $\left\{V_{n}(x): x \in C_{n}\right\}$ is a Borel partition of 
$\mathbb{T}^{k}$. Since the $n / 2$-balls around points in $C_{n}$ are pairwise disjoint, every set $V_{n}(x)$ contains a set of the form $F_{n}\left(x^{\prime}, a_{\mathbf{u}}\right)$ (where $x^{\prime}$ is a point of distance $n / 2$ from $x$ ).

Next, we compute an upper bound on the size of $\partial V_{n}(x)$. Fix $x \in X$. The $3 n$-ball around $x$ has size $(6 n+1)^{d} \leq(7 n)^{d}$. Since the $n / 2$-balls around points in $B$ are disjoint and have size $(n+1)^{d} \geq n^{d}$, there are at most $(7 n)^{d} / n^{d}=7^{d}$ many points $y$ of distance $\leq 2 n$ from $x$, since the $n / 2$-ball around $y$ must be contained in the $3 n$-ball around $x$. Now given any two points $x, y \in B$ such that $d(x, y) \leq 2 n$, the set of $z$ such that $d(x, z)=d(y, z) \leq n$ has size $O\left(n^{(d-1)}\right)$. Since there are at most $7^{d}$ such $y \in B$ in the $2 n$-ball around $x$, the boundary of $V_{n}(x)$ has size $O\left(n^{(d-1)}\right)$. Thus, we can find some $K$ such that for every $x \in X$,

$$
c\left|\partial V_{K}(x)\right| \leq\left|V_{K}(x) \cap A\right| \quad \text { and } \quad c\left|\partial V_{K}(x)\right| \leq\left|V_{K}(x) \cap B\right|
$$

Now finish as before using these Voronoi cells $\left\{V_{K}(x): x \in C_{K}\right\}$ instead of the Gao-Jackson tiling.

\section{The Borel COMPlexity of OUR Equidecompositions}

In this section, we make some remarks about the complexity of the Borel pieces used in the proof of Theorem 1.2. The task of computing these complexities is standard, and we merely sketch a outline of the argument to show the pieces are $\mathcal{B}_{4}^{A, B}$. Fix $A, B \subseteq \mathbb{T}^{k}$ and the action $a_{\mathbf{u}}: \mathbb{Z}^{d} \curvearrowright \mathbb{T}^{k}$ from the proof of Theorem 1.2 We begin with a remark we will use several times when computing complexities.

Remark 7.1. Suppose $C \subseteq \mathbb{T}^{k}$ is defined in terms of some sets $D_{1}, \ldots, D_{n} \subseteq \mathbb{T}^{k}$. If there is some $m$ and a deterministic algorithm which decides if $x \in C$ based on inspecting what vertices of the $m$-ball around $x$ in the graph $G_{a_{\mathbf{u}}}$ lie in $D_{1}, \ldots, D_{n}$, then $C$ is a finite boolean combination of the sets $g \cdot D_{i}$ where $|g|_{\infty} \leq k$ and $1 \leq i \leq n$. Hence, if $D_{1}, \ldots, D_{n} \in \mathcal{B}_{m}^{A, B}$, then we also have $C \in \mathcal{B}_{m}^{A, B}$.

In the cases where we apply Remark 7.1 to compute complexities, such an algorithm will be clear from the proof where we construct the corresponding set.

We now proceed to calculate the complexity of the sets at each step in our argument. Recall from Section 4 that

$$
\psi(x, \gamma \cdot x)=\sum_{n>0} \frac{1}{2^{n d}} \sum_{h \in \mathbb{Z}^{d} /\left(2^{n} \mathbb{Z}\right)^{d}} \phi_{x, h}(x, \gamma \cdot x)-\phi_{x, h}(x, \gamma \cdot x) .
$$

Let $\psi_{k}(x, \gamma \cdot x)$ be the first $k$ many terms of this summation, so

$$
\psi_{k}(x, \gamma \cdot x)=\sum_{n=1}^{k} \frac{1}{2^{n d}} \sum_{h \in \mathbb{Z}^{d} /\left(2^{n} \mathbb{Z}\right)^{d}} \phi_{x, h}(x, \gamma \cdot x)-\phi_{x, h}(x, \gamma \cdot x) .
$$

Then it is clear that $\psi_{k}$ can take only finitely many rational values, and for each $\gamma \in \mathbb{Z}^{d}$ with $|\gamma|_{\infty}=1$, and every possible value $a$, by Remark 7.1 .

$$
\left\{x: \psi_{k}(x, \gamma \cdot x)=a\right\} \in \mathcal{B}_{1}^{A, B} .
$$

Define

$$
\epsilon_{k}=\frac{1}{2^{d-1}} \sum_{n=k-1}^{\infty} \frac{\Phi\left(2^{n}\right)}{2^{n(d-1)}}
$$


to be the tails of the summation defining the constant $c$ in Lemma 4.2, where $\Phi$ is as in the proof of Theorem 1.2 Then $\left|\psi_{k}(x, y)-\psi(x, y)\right|<\epsilon_{k}$ for every edge $(x, y)$ in $G_{a_{\mathrm{u}}}$. Hence, for each real number $a$,

$$
\{x: \psi(x, \gamma \cdot x)<a\} \in \mathbf{\Sigma}_{2}^{A, B}
$$

since $x$ is in this set if and only if $\psi_{k}(x, \gamma \cdot x)+\epsilon_{k}<a$ for some $k$. Indeed, for any finite sequence $\left(g_{1}, \gamma_{1}\right), \ldots,\left(g_{n}, \gamma_{n}\right)$, where $g_{i}, \gamma_{i} \in \mathbb{Z}^{d}$ and $\left|\gamma_{i}\right|_{\infty}=1$, we have that

$$
\left\{x:\left(\sum_{i=1}^{n} \psi\left(g_{i} \cdot x, \gamma_{i} \cdot\left(g_{i} \cdot x\right)\right)\right)<a\right\} \in \boldsymbol{\Sigma}_{2}^{A, B}
$$

by the same argument.

In Section 2, we discussed how at some points in our proof, we use a Borel linear ordering on $\mathbb{T}^{k}$ to make an arbitrary choice in our construction. In order to obtain pieces in our decomposition with the lowest possible complexity, we need to use a particular ordering. Since the comparisons we make in our proof are only between points in the same orbit of $a_{\mathbf{u}}$, it is enough to have a Borel partial order which is linear on each orbit. So given $x, g \cdot x$ in the same orbit of $a_{\mathbf{u}}$, define $x<g \cdot x$ if $g$ is greater than 0 in the lexicographic ordering on elements of $\mathbb{Z}^{d}$. We use this ordering because it combines well with Remark 7.1 .

Next, we consider the proof of Theorem 5.5 in Appendix A. The maximal $r_{i^{-}}$ discrete sets constructed after our statement of Theorem A.1 are $\mathcal{B}_{1}^{A, B}$. It is straightforward to see that the sets $C_{i}$ constructed by Lemma A.2 are each $\mathcal{B}_{3}^{A, B}$; the complicated part of their definition is finding the least $g$ such that $D^{*}(x) \cap\left(g+[0,1]^{d}\right)$ is nonempty. Hence, for the sets $D_{i}$ constructed in the proof of Theorem [5.5, for any $g_{1}, \ldots, g_{n} \in \mathbb{Z}^{d}$,

$$
\left\{x \in \mathbb{T}^{k}:\left\{g_{1} \cdot x, \ldots, g_{n} \cdot x\right\} \in D_{i}\right\} \in \mathcal{B}_{3}^{A, B}
$$

by Remark 7.1. Note that for each $i$, there is an upper bound on the size of all elements of $D_{i}$.

Now consider Lemma 5.4 where we turn our real-valued flow $\psi$ into an integral Borel flow, which we will call $\psi^{\prime}$. (Our variable choices here differ from the $\phi$ and $\psi$ in the statement of Lemma 5.4). We claim that for each $i$, letting $D_{i}$ be as in the proof of Theorem 5.5 as above, for every $m$,

$$
\left\{x \in \mathbb{T}^{k}: x \in \bigcup D_{i} \wedge \psi^{\prime}(x, \gamma \cdot x)=m\right\} \in \mathcal{B}_{3}^{A, B} .
$$

This is by combining the fact that for each $i$, the elements of $D_{i}$ have bounded size with the fact proved above that $\left\{x:\left(\sum_{i=1}^{n} \psi\left(g_{i} \cdot x, \gamma_{i} \cdot\left(g_{i} \cdot x\right)\right)\right)<a\right\} \in \boldsymbol{\Sigma}_{2}^{A, B}$, and then using Remark 7.1. Thus, taking the union of these $\mathcal{B}_{3}^{A, B}$ sets, we see that for every $m$,

$$
\left\{x \in \mathbb{T}^{k}: \psi^{\prime}(x, \gamma \cdot x)=m\right\} \in \boldsymbol{\Sigma}_{4}^{A, B} .
$$

in our integral Borel flow $\psi^{\prime}$.

Finally, consider the argument in Section 6 which uses Remark 6.2 to define the equidecomposition. Since our equidecomposition just uses the value of $\psi^{\prime}$ (whose complexity has been computed above), and a maximal $K$-discrete set which is $\mathcal{B}_{1}^{A, B}$, the resulting pieces will be $\mathcal{B}_{4}^{A, B}$ by Remark 7.1 (Similarly, inspecting the proof of Lemma 6.1 in [GJ] also yields pieces with the same complexity at this last step). 


\section{A. Proof of Theorem 5.5}

In this appendix, we give a proof of Theorem 5.5. If $d$ is a metric on a set $X$, then we say that $Y \subseteq X$ is $r$-discrete (with respect to $d$ ) if $d(x, y)>r$ for all distinct $x, y \subseteq Y$. We further say that $Y \subseteq X$ is a maximal $r$-discrete set if $Y$ is $r$-discrete and for every $x \in X$ there is a $y \in Y$ with $d(x, y) \leq r$. If $G$ is a graph on $X$ then by an $r$-discrete set of vertices in $X$, we mean with respect to the graph metric $G$ induces on $X$.

We will need the following theorem of Kechris, Solecki, and Todorcevic.

Theorem A.1 ([KST, Theorem 4.2]). Every locally finite Borel graph G has a maximal $r$-discrete Borel set.

In the specific case of the graph $G_{a_{\mathbf{u}}}$ we can give a short proof of this fact. Given any $r>0$, for sufficiently small $\epsilon>0$, the $\epsilon$-ball around any point $x \in \mathbb{T}^{k}$ will be an $r$-discrete Borel set. Thus, we can find a maximal $r$-discrete Borel set $C$ for $G_{a_{\mathrm{u}}}$ as follows. Let $B_{0}, B_{1}, \ldots, B_{n}$ be finitely many $\epsilon$-balls which cover $\mathbb{T}^{k}$. Define $C_{0}=B_{0}$ and let $C_{i+1}=B_{i+1} \backslash \bigcup\left\{g \cdot C_{j}: j \leq i \wedge|g|_{\infty} \leq r\right\}$. So inductively, $\bigcup_{i \leq k} C_{i}$ is an $r$-discrete set and for every $x \in B_{k}$, there is a $y \in C_{k}$ such that $d(x, y) \leq r$. Now finish by letting $C=\bigcup_{i \leq n} C_{i}$.

Next, we need the following lemma which is a rephrasing of an idea due to Boykin and Jackson. They used it to give a new proof of the theorem originally due to Weiss that free Borel actions of $\mathbb{Z}^{d}$ are hyperfinite.

Lemma A.2 ([B]]). Suppose $d \geq 1, n>0, a: \mathbb{Z}^{d} \curvearrowright X$ is a free Borel action of $\mathbb{Z}^{d}$ on a standard Borel space $X$, and $r_{0}<r_{1}<\ldots$ is an increasing sequence of natural numbers. Then there is a sequence $C_{0}, C_{1}, \ldots \subseteq X$ of Borel sets such that $C_{i}$ is Borel maximal $r_{i}$-discrete set for $G_{a}$, and for every $\epsilon>0$ and every $x \in X$, there are infinitely many $i$ such that $d\left(x, C_{i}\right)<\epsilon r_{i}$.

Proof. For each $i$, by Theorem A.1, let $C_{i}^{\prime}$ be a Borel maximal $r_{i}$-discrete set for the graph $G_{a}$. To each $x \in X$, we associate a sequence $D_{0}(x), D_{1}(x) \ldots$ of subsets of $\mathbb{R}^{d}$. Let

$$
D_{i}(x)=\left\{g / r_{i}: g \in \mathbb{Z}^{d} \wedge(-g) \cdot x \in C_{i}^{\prime}\right\}
$$

So each set $D_{i}(x)$ is a maximal 1-discrete subset of $\mathbb{R}^{d}$, with respect to metric $|\cdot|_{\infty}$ on $\mathbb{R}^{d}$. Now let $D^{*}(x) \subseteq \mathbb{R}^{d}$ be the set of accumulation points of the sequence $D_{0}(x), D_{1}(x), \ldots$, so $D^{*}(x)$ is a closed subset of $\mathbb{R}^{d}$. $D^{*}(x)$ is nonempty since each set $D_{i}(x)$ contains at least one point in the set $[0,1]^{d}$ which is compact. Now if $x, y \in X$ are in the same orbit of $a$, then $D^{*}(x)=D^{*}(y)$, since the set $D_{i}(x)$ can be shifted by a distance of $d(x, y) / r_{i}$ to become equal to $D_{i}(y)$, and $d(x, y) / r_{i} \rightarrow 0$ as $i \rightarrow \infty$.

Recall that a function $f$ on $X$ is said to be $a$-invariant if $f(x)=f(y)$ for all $x, y$ in the same $a$-orbit of $X$. We claim that there is an $a$-invariant Borel function $f: X \rightarrow$ $\mathbb{R}^{d}$ such that $f(x) \in D^{*}(x)$ for every $x \in T^{k}$. Let $f_{n}(x)$ be the lexicographically least $g \in\left\{0, \ldots, r_{n}-1\right\}^{d}$ such that $D^{*}(x) \cap\left(g+[0,1]^{d}\right) / r_{n}$ is nonempty. Then each function $f_{n}$ is $a$-invariant, and by the definition of the lexicographic order, the lexicographically least element of $D^{*}(x) \cap[0,1]^{d}$ is contained in $\left(f_{n}(x)+[0,1]^{d}\right) / r_{n}$. If we let $f(x)=\lim _{n \rightarrow \infty} f_{n}(x) / r_{i}$, then $f(x)$ is the lexicographically least element of $D^{*}(x) \cap[0,1]^{d}$, and $\left|f(x)-f_{i}(x)\right|_{\infty}<1 / r_{i}$ for every $i$. Note that $f$ is $a$-invariant.

For every $x \in X$ and $\epsilon>0$ there are infinitely many $i$ such that $d\left(f(x), D_{i}(x)\right)<$ $\epsilon / 2$, since $f(x)$ is an accumulation point of the $D_{i}(x)$. Hence, there are infinitely 
many $i$ such that $d\left(f_{i}(x) / r_{i}, D_{i}(x)\right)<\epsilon / 2+1 / r_{i}$. Using the definition of $D_{i}(x)$, this means there are infinitely many $i$ such that there exists some $y \in C_{i}^{\prime}$ such that $d\left(\left(-f_{i}(x)\right) \cdot x, y\right)<\epsilon r_{i} / 2+1$.

Now if we define

$$
C_{i}=\left\{f_{i}(y) \cdot y: y \in C_{i}^{\prime}\right\}
$$

then using the $a$-invariance of $f_{i}$, we see that there are infinitely many $i$ such that $d\left(x, C_{i}\right)<\epsilon r_{i} / 2+1$ which suffices to prove the theorem.

It seems likely that one can prove an analogous result for any finitely generated nilpotent group $\Gamma$ instead of $\mathbb{Z}^{d}$ by using the Mal'cev completion of $\Gamma$ in place of $\mathbb{R}^{d}$ in the argument above. However, the analogous problem for arbitrary finitely generated amenable groups is open. A positive answer would imply that every free Borel action of a finitely generated amenable group is hyperfinite, which is a well-known open problem (see [JKL, GJ], and [SS]).

Problem A.3. Let $\Gamma$ be a finitely generated amenable group with symmetric generating set $S$, and $a: \Gamma \curvearrowright X$ be a free Borel action of $\Gamma$ on a standard Borel space $X$. Let $G_{a, S}$ be the Borel graph on $X$ where there is an edge between $x$ and $y$ if there exists a $\gamma \in S$ such that $\gamma \cdot x=y$. Must it be the case that for every increasing sequence $r_{0}<r_{1}<\ldots$, there is a sequence $C_{0}, C_{1}, \ldots$ of Borel subsets of $X$ such that $C_{i}$ is a Borel maximal $r_{i}$-discrete set, and for every $\epsilon>0$ and $x \in X$, there are infinitely many $i$ such that $d_{G_{a, S}}\left(x, C_{i}\right)<\epsilon r_{i}$ ?

Lemma A.2 will be used in our proof of Lemma 5.5 to ensure that the elements of $C$ cover $X$. The next definition and lemma will be used to ensure that elements of $C$ have disjoint boundaries.

Definition A.4. Suppose $S$ is a set of vertices in a graph $G$ on $X$. Then let $B_{r}(S)=\{x: d(x, S) \leq r\}$ be the "ball" of radius $r$ around $S$. Abusing notation, if $Y \subseteq[G]^{<\infty}$, let $B_{r}(Y)=\left\{B_{r}(S): S \in Y\right\}$. Finally, if $Y, Z \subseteq[G]^{<\infty}$, let

$$
B_{r}(Y, Z)=\left\{S \cup \bigcup\left\{B_{r}(R): R \in Z \wedge d(R, S) \leq r\right\}: S \in Y\right\} .
$$

That is, for each $S \in Y, B_{r}(Y, Z)$ contains the set consisting of $S$ together with the $r$-balls around all elements of $Z$ of distance at most $r$ from $S$.

We have the following triviality

Lemma A.5. Suppose $Y, Z \subseteq[G]<\infty$ are such that $\operatorname{diam}(R) \leq r$ for every $R \in Z$, for all distinct $R, R^{\prime} \in Z, d\left(R, R^{\prime}\right)>2 r$, and $d\left(S, S^{\prime}\right)>6 r$ for every $S, S^{\prime} \in Y$. Then

(1) For every $Q \in B_{r}(Y, Z)$ there is an $S \in Y$ such that $S \subseteq Q \subseteq B_{3 r}(S)$.

(2) Every element of $B_{r}(Y, Z)$ is finite and connected, and the elements of $B_{r}(Y, Z)$ are pairwise disjoint.

(3) If $R \in Z$ and $Q \in B_{r}(Y, Z)$, then either $B_{r}(R) \subseteq Q$, or $d(R, Q)>r$.

We now use Lemma A.2 to prove Theorem 5.5 .

Proof of Theorem 5.5. Let $r_{i}=n 12^{i+1}$, and let $C_{0}, C_{1}, \ldots \subseteq X$ be Borel maximal $4 r_{i}$-discrete sets satisfying the conclusion of Lemma A.2. We now define sets $D_{i} \subseteq$ $[G]^{<\infty}$. Given that we have defined $D_{j}$ for $j<i$, we define $D_{i}$ by constructing a sequence $A_{0}^{i}, \ldots, A_{i}^{i} \subseteq[G]^{<\infty}$, and letting $D_{i}=A_{i}^{i}$ at the end. To begin, let 
$A_{0}^{i}=B_{r_{i} / 4}\left(C_{i}\right)$. Hence, by the definition of $C_{i}$, the elements of $A_{0}^{i}$ are connected, have diameter $\leq r_{i} / 2$, and for all distinct $R, R^{\prime} \in A_{0}^{i}$ we have $d\left(R, R^{\prime}\right)>3 r_{i}$.

For $0<j \leq i$, let

$$
A_{j}^{i}=B_{r_{i-j}}\left(A_{j-1}^{i}, D_{i-j}\right) .
$$

We claim that at the end of this construction, the elements of $D_{i}=A_{i}^{i}$ have diameter $\leq r_{i}$ and are pairwise of distance at least $2 r_{i}$. This is easy to prove by induction using Lemma A.5. since for every $Q \in D_{i}=A_{i}^{i}$, there is some $S \in A_{0}^{i}$ such that

$$
Q \subseteq B_{3 r_{0}}\left(\ldots B_{3 r_{i-2}}\left(B_{3 r_{i-1}}(S)\right) \ldots\right)
$$

and so $\operatorname{diam}(Q) \leq r_{i} / 2+6 r_{i-1}+\ldots+6 r_{0}$ which is at most $r_{i}$ by our definition of $r_{i}$. Next, note that for each $0<j \leq i$, if $R \in D_{i-j}$ and $Q \in D_{i}$, then by induction using Lemma A.5 either $B_{r_{i-j}}(R) \subseteq Q$, or $d(Q, R)>r_{i-j}-3 r_{i-j-1}-\ldots-3 r_{0}$. Note that $r_{i-j}-3 r_{i-j-1}-\ldots-3 r_{0} \geq r_{0}$ for all $0<j \leq i$.

To finish, let $C=\left\{\tilde{S}: S \in \bigcup_{i} D_{i}\right\}$, where $\tilde{S}$ is the set of vertices in $S$ together with all $x \in X$ that are in some finite connected component of $G-\partial S$. It is clear that $\partial \tilde{S} \subseteq \partial S$, and that $\partial_{\text {vis }(\infty)} \tilde{S}=\partial \tilde{S}$ as described in the paragraph after the statement of Theorem [5.5. It follows from the previous paragraph that all distinct $R, S \in \bigcup_{i} D_{i}$ have $\partial^{n} R \cap \partial^{n} S=\emptyset$, since $r_{0}>2 n$. Finally, $\bigcup C=X$ since $\bigcup\left(\bigcup_{i} A_{0}^{i}\right)=X$ by our choice of the sequence $C_{i}$.

\section{REFERENCES}

[ABGPS] R. Aharoni, E. Berger, A. Georgakopoulos, A. Perlstein, and P. Sprüssel, The max-flow min-cut theorem for countable networks, J. Combin. Theory Ser. B 101 (2011), no. 1, 1-17.

[BJ] C. Boykin and S. Jackson, Borel boundedness and the lattice rounding property, Advances in logic, 113126, Contemp. Math., 425, Amer. Math. Soc., Providence, RI, 2007.

[D] R. Diestel, Graph theory. Fourth edition. Graduate Texts in Mathematics, 173. Springer, Heidelberg.

[DHK] L. Dubins, M. W. Hirsch, and J. Karush, Scissor congruence, Israel J. Math. 1 (1963), 239-247.

[G] R. J. Gardner, Convex bodies equidecomposable by locally discrete groups of isometries, Mathematika 32 (1985), 1-9.

[GJ] S. Gao and S. Jackson, Countable abelian group actions and hyperfinite equivalence relations. Invent. Math. 201 (2015), no. 1, 309-383.

[GJKS] S. Gao, S. Jackson, E. Krohne, and B. Seward Forcing constructions and countable Borel equivalence relations. Preprint. 2015. http://www.math.unt.edu/ sgao/pub/pub.htm

[GMP] L. Grabowski, A. Máthé, O. Pikhurko, Measurable circle squaring, arXiv:1501.06122v3.

[JKL] S. Jackson, A.S. Kechris, and A. Louveau, countable Borel equivalence relations, J. Math. Logic, 2 (2002), 1-80.

[NW] H. Niederreiter and J. M. Wills, Diskrepanz und Distanz von Massen besüglich konvexer und Jordanscher Mengen, Math. Z. 144 (1975) 125-134.

[K] A.S. Kechris, Classical descriptive set theory, Springer, 1995.

[KM] A.S. Kechris and A.S. Marks, Descriptive graph combinatorics. Preprint, 2016. http://www.math.caltech.edu/ kechris

[KST] A.S. Kechris, S. Solecki and S. Todorcevic, Borel chromatic numbers, Adv. Math., 141 (1999), 1-44.

[L88] M. Laczkovich, Closed sets without measurable matching, P. Amer. Math. Soc. 103 (1988), no. $3,894-896$.

[L90] M. Laczkovich, Equidecomposability and discrepancy; a solution of Tarski's circle-squaring problem, J. Reine Angew. Math. 404 (1990), 77-117.

[L92] M. Laczkovich, Decomposition of sets with small boundary, J. Lond. Math. Soc. 46 (1992), 58-64. 
[S] W. Schmidt, Metrical theorems on fractional parts of sequences, Trans. Amer. Math. Soc. 110 (1964) 493-518.

[SS] S. Schneider and B. Seward, Locally nilpotent groups and hyperfinite equivalence relations, Preprint.

[T] A. Tarski, Probléme 38, Fund. Math. 7 (1925), 381.

[Ti] Á. Timár, Boundary-connectivity via graph theory, Proc. Amer. Math. Soc. 141 (2013), no. $2,475-480$.

[W] S. Wagon, The Banach Tarski Paradox, University Press, Cambridge, 1986.

Department of Mathematics, University of California at Los Angeles

E-mail address: marks@math.ucla.edu

Department of Mathematics, University of California at Los Angeles

E-mail address: sunger@math.ucla.edu 